\title{
Targeting microRNA/UHRF1 pathways as a novel strategy for cancer therapy (Review)
}

\author{
HANI CHOUDHRY ${ }^{1-4}$, MAZIN A. ZAMZAMI ${ }^{1-3}$, ZIAD OMRAN $^{5}$, WEI WU ${ }^{6}$, \\ MARC MOUSLI $^{7}$, CHRISTIAN BRONNER $^{8}$ and MAHMOUD ALHOSIN ${ }^{1-3}$
}

\author{
${ }^{1}$ Department of Biochemistry, Faculty of Science; ${ }^{2}$ Cancer Metabolism and Epigenetic Unit; ${ }^{3}$ Cancer and Mutagenesis Unit, \\ King Fahd Medical Research Center; ${ }^{4}$ Center of Innovation in Personalized Medicine, King Abdulaziz University, \\ Jeddah 21589; ${ }^{5}$ College of Pharmacy, Umm Al-Qura University, Makkah 21955, Saudi Arabia; ${ }^{6}$ Department \\ of Medicine, University of California, San Francisco, CA 94143, USA; ${ }^{7}$ Laboratory of Biophotonics and \\ Pharmacology, Faculty of Pharmacy, University of Strasbourg, 67401 Illkirch Cedex; ${ }^{2}$ Institute of Genetics and \\ Molecular and Cellular Biology (IGBMC), National Institute of Health and Medical Research U964, National \\ Center for Scientific Research UMR7104, University of Strasbourg, 67404 Illkirch Cedex, France
}

Received June 14, 2017; Accepted September 22, 2017

DOI: $10.3892 / \mathrm{ol} .2017 .7290$

\begin{abstract}
Ubiquitin-like containing plant homeodomain and RING finger domains 1 (UHRF1) is an anti-apoptotic protein involved in the silencing of several tumor suppressor genes (TSGs) through epigenetic modifications including DNA methylation and histone post-translational alterations, and also epigenetic-independent mechanisms. UHRF1 overexpression is observed in a number of solid tumors and hematological malignancies, and is considered a primary mechanism in inhibiting apoptosis. UHRF1 exerts its inhibitory activity on TSGs by binding to functional domains and therefore influences several epigenetic actors including DNA methyltransferase, histone deacetylase 1 , histone acetyltransferase Tat-interacting protein 60 and histone methyltransferases G9a and Suv39H1. UHRF1 is considered to control a large macromolecular protein complex termed epigenetic code replication machinery,
\end{abstract}

Correspondence to: Dr Mahmoud Alhosin, Department of Biochemistry, Faculty of Science, King Abdulaziz University, 90A Al-Marsad Street, Jeddah 21589, Saudi Arabia

E-mail: malhaseen@kau.edu.sa

Abbreviations: UHRF1, ubiquitin-like with PHD and RING finger domains 1; TSG, tumor suppressor gene; HDAC1, histone deacetylase 1; DNMT1, DNA methyltransferase 1; Tip60, Tat-interacting protein 60; HAUSP, herpesvirus-associated ubiquitin-specific protease; TTD, tandem Tudor domain; PHD plant homeodomain; SRA, SET- and RING-associated domain; RING, really interesting new gene; ECREM, epigenetic code replication machinery; 3'-UTR, 3'-untranslated region; CRC, colorectal cancer; BC, bladder cancer; NSCLC, non-small cell lung cancer; GC, gastric cancer

Key words: apoptosis, cancer, DNA methylation, epigenetics, microRNA, tumor suppressor genes, ubiquitin-like with PHD and RING finger domains 1 in order to maintain epigenetic silencing of TSGs during cell division, thus enabling cancer cells to escape apoptosis. MicroRNAs (miRNAs) are able to regulate the expression of its target gene by functioning as either an oncogene or a tumor suppressor. In the present review, the role of tumor suppressive miRNAs in the regulation of UHRF1, and the importance of targeting the microRNA/UHRF1 pathways in order to induce the reactivation of silenced TSGs and subsequent apoptosis are discussed.

\section{Contents}

1. Introduction

2. Role of UHRF1 in carcinogenesis

3. Role of miRNAs in cancer

4. Regulation of UHRF1 by miR-146a/b in gastric cancer

5. Regulation of UHRF1 by miRNAs in bladder cancer and kidney tumors

6. Regulation of UHRF1 by miR-9 in colorectal cancer

7. Regulation of UHRF1 by miR-193a-3p in NSCLC

8. Conclusion

\section{Introduction}

Epigenetic silencing of tumor suppressor genes (TSGs) including breast cancer susceptibility gene 1 (BRCAl), human MutL homolog 1 ( $h M L H 1), p 16^{I N K 4 A}$ and $p 14^{A R F}$ involves DNA methylation maintained by DNA methyltransferase 1 (DNMT1), histone deacetylation and methylation through histone deacetylase 1 (HDAC1) and histone H3K9 methyltransferase G9a respectively (1-3). Ensuring a coordinated crosstalk between DNA methylation and histone deacetylation and methylation, ubiquitin-like containing plant homeodomain (PHD) and really interesting new gene domain (RING) finger domains 1 (UHRF1) overexpressed in various human 
cancer cells induces epigenetic silencing in several TSGs (4). UHRF1-mediated epigenetic silencing of TSG is primarily due to the presence of the SET- and RING-associated (SRA) domain (4). Through the SRA domain, UHRF1 interacts with HDAC1 and DNMT1 (Fig. 1), leading to the inhibition of several TSGs including $p 16^{I N K 4 A}, p 14^{A R F}$ and retinoic acid receptor $\alpha(1,3,5,6)$. Furthermore, the UHRF1 structure includes other functional domains which contribute to its inhibitory activity on TSGs including the ubiquitin-like domain, tandem Tudor domain (TTD), PHD and RING domain (Fig. 1). A large macromolecular protein complex termed epigenetic code replication machinery (ECREM) is formed through interactions between the different UHRF1 domains and several epigenetic coordinators including HDAC1, DNMT1, histone acetyltransferase, Tat-interacting protein 60 (Tip60), herpesvirus-associated ubiquitin specific protease (HAUSP) and histone methyltransferase G9a and Suv39H1 (Fig. 1) (7-9).

The ECREM complex is considered to be orchestrated by UHRF1 to ensure a coordinated transmission of silenced TSGs to daughter cells during cell division $(4,7,9)$. UHRF1 binds to $\mathrm{H} 3 \mathrm{~K} 9 \mathrm{me}$, a repressive chromatin mark, thus providing additional evidence of UHRF1-mediated crosstalk between DNA methylation and histone modification (10). Furthermore, UHRF1 was also demonstrated to bind to H3K9me3 through the TTD domain, an interaction involved in the regulation of p16 ${ }^{\mathrm{INK} 4 \mathrm{~A}}$ expression (11). Although overexpression of wild-type UHRF1 induced $p 16^{I N K 4 A}$ downregulation, such an effect was not demonstrated when the TTD-mutated UHRF1 variant was overexpressed, thus indicating that UHRF1 binding to $\mathrm{H} 3 \mathrm{~K} 9 \mathrm{me} 3$ through the TTD domain is involved in the silencing of $p 16^{I N K 4 A}(11)$. In the same context, UHRF1 was demonstrated to use its PHD domain to specifically bind to H3K9me3 (12) and cause large-scale modifications of chromocenters which assisted in the recruitment of HDAC1 and DNMT1, and led to the formation of pericentromeric heterochromatin (13). The UHRF1, through its RING domain, was demonstrated to possess E3 ubiquitin ligase activity for histone 3 , and was also involved in tumor proliferation; however, the role of this domain remains unclear $(14,15)$. UHRF1 interacts with HAUSP (Fig. 1), a deubiquitinating enzyme involved in the regulation of several TSGs (16). HAUSP protects UHRF1 from its own E3 ligase activity (autoubiquitination), suggesting that UHRF1 uses its RING domain to target itself for degradation via autoubiquitination in response to the downregulation of HAUSP (17-19).

Collectively, these previous studies demonstrate that UHRF1 through its several functional domains negatively regulates the expression of TSGs via its interaction with numerous proteins. Thus, the inhibition of the expression and/or activity of UHRF1 may enable cancer cells to undergo apoptosis by reactivating TSGs. As UHRF1 belongs to a large macromolecular complex, in which it serves a function of a hub protein for the integration of epigenetic information, microRNAs (miRNAs) which target UHRF1 may have marked effects on cellular functions. In the present review, the role of these specific miRNAs in the regulation of UHRF1 (Table I), and the associated downstream events, as well as the importance of targeting miRNA/UHRF1 pathways as a novel strategy in cancer therapy, are discussed.

\section{Role of UHRF1 in carcinogenesis}

Several previous studies indicate that UHRF1 may be a key regulator of the human epigenome through interactions with domains in several types of coordinator (7,20-23). These interactions indicate that UHRF1 is involved in carcinogenesis through two key mechanisms. The first is regarded as a participation in the onset phase of cancer, whereas the second is with regard to the maintenance of the cancer phenotype. Concerning the first, the driving of DNA hypomethylation is a result of defective DNMT1-UHRF1 interaction $(24,25)$. The second is associated with the maintenance of DNA methylation patterns, particularly the hypermethylation of the TSG promoters and genome-wide hypomethylation (6).

Although the role of UHRF1 as a potent oncogene is well-documented in several solid tumors and hematological malignancies, its downregulation has been demonstrated to increase the malignancy of carcinoma cells through the activation of epithelial-mesenchymal transition (EMT) (3,4,26-35). Numerous types of human cancer including leukemia, breast, bladder, gastric, colorectal and astrocytoma express increased levels of UHRF1, causing an increase in cell proliferation, migration, metastasis and inhibition of apoptosis (7,36-39). The UHRF1 serves a crucial function in the progression of the cell cycle at $\mathrm{G}_{1} / \mathrm{S}$ phase through the $p 16^{I N K 4 A}$-dependent pathway, and its downregulation allows cancer cells to undergo apoptosis through DNA demethylation and histone deacetylation-dependent reactivation of several TSGs including pl $^{I N K 4 A}, B R C A 1$, homeobox protein CDX-2 (CDX2), runt-related transcription factor 3 (RUNX3), forkhead box protein $\mathrm{O} 4$, peroxisome-proliferator-activated receptor $\gamma$ and promyelocytic leukemia $(P M L)(2,32,40)$. UHRF1 overexpression in cancer cells, compared with matched normal tissue was suggested to be a potential biomarker for the prognosis and diagnosis of several types of cancer including bladder and colorectal carcinoma $(23,36,39)$. Considering that cancer cells overexpress UHRF1, deciphering the upstream pathways involved in UHRF1 may shed light on the underlying molecular mechanisms involved in the silencing of TSGs in tumorigenesis.

\section{Role of miRNAs in cancer}

High-throughput transcriptome analysis demonstrated that the majority of transcriptional outcome is non-coding RNAs (41). These non-coding RNAs are classified, based on their length, into small non-coding RNAs ( $<200$ nucleotides), including miRNA, and long non-coding RNA (>200 nucleotides), including nuclear paraspeckle assembly transcript $1(42,43)$. A number of non-coding RNAs have potential transcriptional, post-transcriptional and epigenetic regulatory functions, and are often dysregulated in many types of disease including cancer (44-47). miRNAs (18-25 nucleotides) are the most studied class of non-coding RNAs and post-transcriptionally regulate mRNA stability and translation (48). The biogenesis of miRNA and the mechanism by which they degrade mRNA and inhibit RNA translation is complex (49). Altered miRNA expression has been observed in many types of cancer cell line, xenograft, blood and clinical tissue $(50,51)$. Aberrant levels of miRNAs contribute to cancer formation and progression by 
Table I. Types of miRNA involved in UHRF1 regulation in various tumors and UHRF1-regulated genes.

\begin{tabular}{lcccc}
\hline miRNA & Type of cancer & Function & Reported target genes of UHRF1 & (Refs.) \\
\hline $146 \mathrm{a} / \mathrm{b}$ & GC & TSG & TSGs: Slit3, CDH4 and RUNX3 & $(67,69)$ \\
124 & BC & TSG & & $(74)$ \\
$145-5 \mathrm{p}, 145-3 \mathrm{p}$ & BC & TSG & Oncogenes: BIRC5 and CENPF & $(75)$ \\
$146 \mathrm{a}-5 \mathrm{p}$ & ccRCC & TSG & TSGs: p53? \\
$193 \mathrm{a}-3 \mathrm{p}$ & NSCLC & TSG & TSGs & $(78)$ \\
101 & RCC & TSG & & $(93,95)$ \\
9 & CRC & TSG & & $(84)$ \\
\hline
\end{tabular}

Question marks indicate putative effects that require further confirmation. miRNA, microRNA; GC, gastric cancer; BC, bladder cancer; ccRCC, clear cell renal cell carcinoma; RCC, renal cell carcinoma; NSCLC, non-small cell lung cancer; TSG, tumor suppressor gene; Slit3, slit homolog 3 protein; CHD4, cadherin 4; RUNX3, runt-related transcription factor 3; BIRC5, survivin; CENPF, centromere protein F.

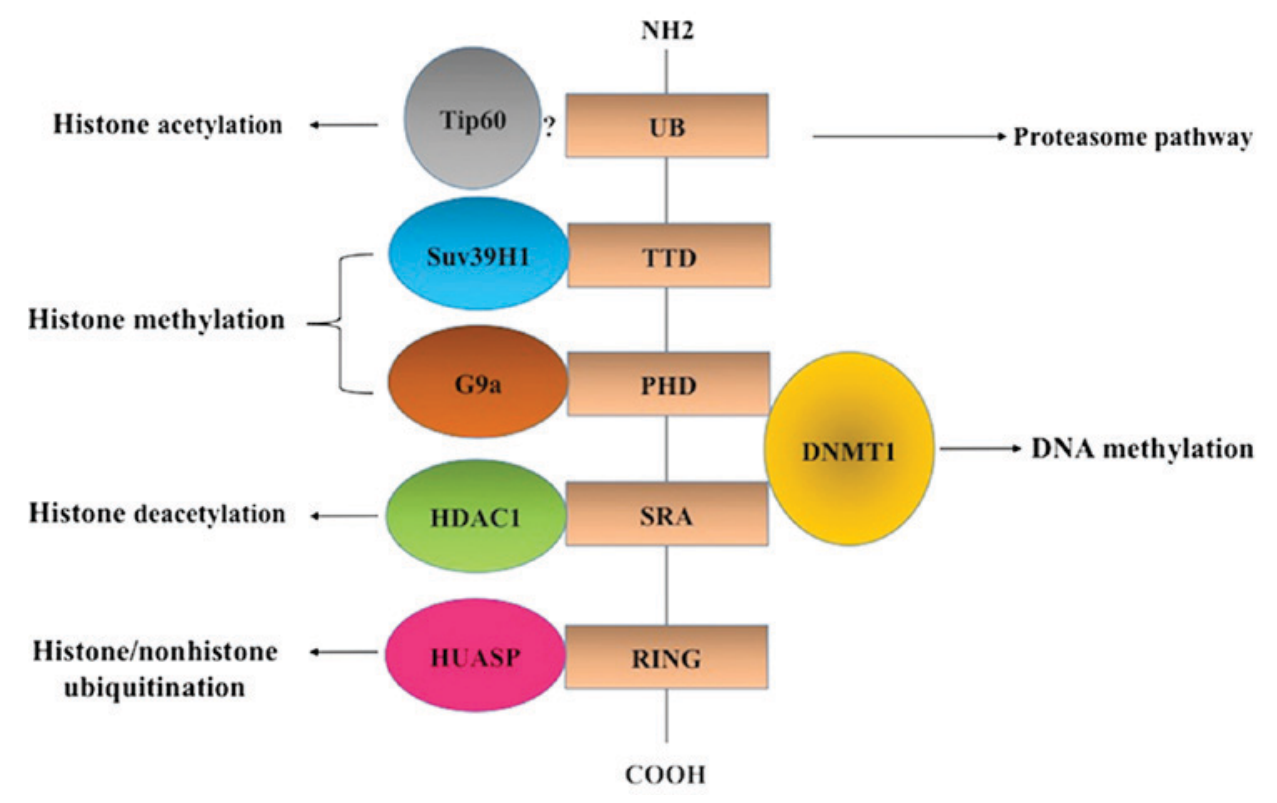

Figure 1. Schematic model of UHRF1 structure and its role in the regulation of the epigenetic code (DNA methylation and histone modifications). Through its SRA domain, UHRF1 interacts with DNMT1 and HDAC1. Using its PHD domain, UHRF1 may interact with the histone methyltransferase G9a and also with DNMT1. UHRF1 may be autoubiquitinated by its RING domain, encompassing an E3 ligase activity. By its interaction with HAUSP, UHRF1 may be protected from autoubiquitination. The TTD is involved in histone methylation reading with the subsequent recruitment of the histone methyltransferases G9a and Suv39H1. The UBL domain may be involved in the proteasome pathway. The question mark represents a putative interaction that requires further confirmation. SRA, SET- and RING-associated; DNMT1, DNA methyltransferase 1; HDAC1, histone deacetylase 1; PHD, plant homeodomain; RING, really interesting new gene; UHRF1, ubiquitin-like with PHD and RING finger domains 1; HUASP, herpesvirus-associated ubiquitin-specific protease; TTD, tandem Tudor domain; UBL, ubiquitin-like; Tip60, Tat-interacting protein 60; UB, ubiquitin.

regulating expression levels of key genes involved in tumorigenesis pathways which are responsible for cell proliferation, tumor migration, invasion, integrin-mediated adhesion, EMT and resistance to cancer therapy (52). Similar to protein-coding genes, miRNAs are also subject to epigenetic regulatory modifications in cancer $(53,54)$. The majority of miRNA loci are associated with $\mathrm{CpG}$ islands suggesting marked dependence on DNA methylation.

In cancer, miRNAs are able to function as being either oncogenic or a tumor suppressor, depending on the target gene (55). For example, miR-21 was the first miRNA to be identified as being oncogenic, and was demonstrated to be overexpressed in numerous types of cancer (56). Mechanistically, miR-21 was observed to suppress the expression of many TSGs, including phosphatase and tensin homolog, programmed cell death protein 4 and sprout 1 (SPRY1) (57,58). However, miR-34b, miR-199b and miR-218 are examples of tumor suppressor miRNAs that were observed to be downregulated in several types of tumor $(59,60)$. Advances in genomic technologies may lead to identification of novel miRNAs involved in cancer, therefore the increase in the understanding of their biological functions and target genes is expected to enhance our knowledge on the role of miRNA in cancer progression and permit the development of miRNA-associated cancer biomarkers and consequently the formation of effective therapy.

Considering the fact that numerous types of human cancer express increased levels of the oncogene UHRF1 in 
$\mathbf{A}$

Gastric cancer (GC)

B

Bladder cancer (BC)

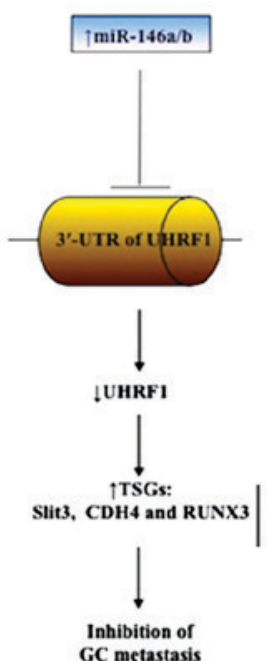

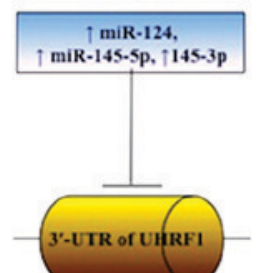

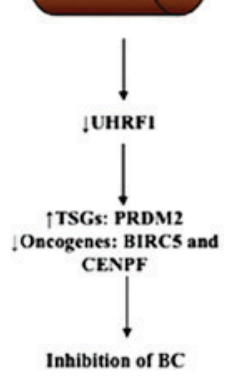

C

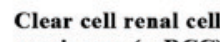
carcinoma (ccRCC)
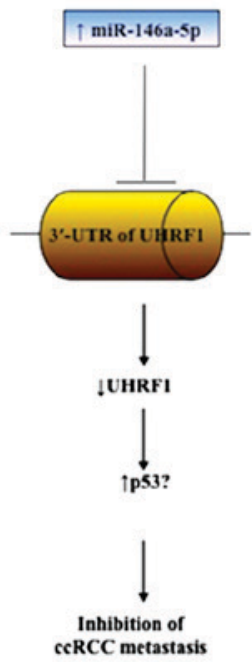

D

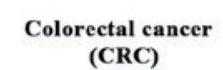

$\mathbf{E}$

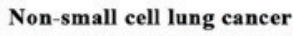

(NSCLC)
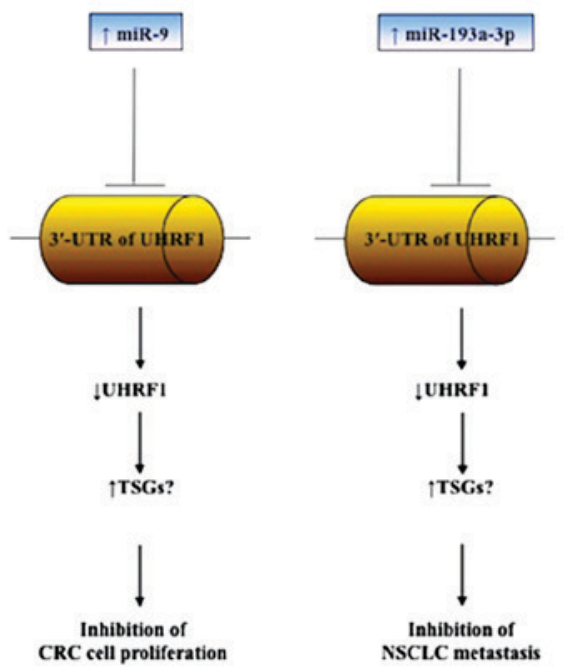

Figure 2. Schematic representation of the role of miRNA in UHRF1 regulation in different types of cancer cell: (A) Gastric cancer, (B) bladder cancer, (C) clear cell renal cell carcinoma, (D) colorectal cancer and (E) non-small cell lung cancer. Several tumor suppressor miRNAs are able to bind to the 3 '-UTR of UHRF1 mRNA. This binding induces UHRF1 mRNA degradation and leads to the reactivation of several TSGs including Slit3, CDH4, RUNX3, PRDM2 and TP53 or to the inhibition of the expression of certain oncogenes including BIRC5 and CENPF. Question marks indicate putative effects that require further confirmation. UHRF1, ubiquitin-like with PHD and RING finger domains 1; TSG, tumor suppressor gene; Slit3, slit homolog 3 protein; CHD4, cadherin 4; RUNX3, runt-related transcription factor 3; PRDM2, PR domain zinc finger protein 2; BIRC5, survivin; CENPF, centromere protein F; miR, microRNA.

association with decreased expression levels of several tumor suppressor miRNAs, UHRF1 overexpression in cancer as a result of altered miRNA expression is a subject worth investigating (36). Nevertheless, it has previously been indicated that UHRF1 overexpression may also be a result of increased stability, and of the inhibitory effects of several miRNAs on its expression (36). However, it should be noted that UHRF1 has been demonstrated to be regulated by several other pathways including the cluster of differentiation 47/nuclear factor $\kappa \mathrm{B}$ axis, TSG p53 and p73, and the thyroid hormone receptor $\alpha 1 /$ specificity protein 1 pathway (61-64)

\section{Regulation of UHRF1 by miR-146a/b in gastric cancer}

Several previous studies have demonstrated that UHRF1 is overexpressed in gastric cancer (GC) and therefore promote the invasion and metastasis of this type of cancer; however, the upstream regulatory mechanisms involved in UHRF1 overexpression are currently unknown $(32,65,66)$. Increased levels of UHRF1 expression were identified in tissue samples from patients with GC compared with normal controls, and its overexpression was associated with patient age and lymph node metastasis (66). The levels of UHRF1 were also significantly increased in tissues isolated from patients with GC compared with corresponding normal tissues. Furthermore, increased expression levels of UHRF1 were identified in GC tissues in association with GC stage and grade (65). Similarly, UHRF1 was demonstrated to be overexpressed in GC, and its downregulation induced upregulation of several TSGs including RUNX3, BRCAl and PML through a promoter demethylation-dependent mechanism, inhibiting GC cell proliferation and metastasis (32). Furthermore, another study demonstrated that UHRF1 was overexpressed in tissues from patients with GC compared with matched normal tissues, and its increased expression levels were associated with GC metastases (67). Of note, UHRF1 depletion decreased GC migration and metastasis; however, overexpression significantly promoted these effects (67). Collectively, the results of these previous studies demonstrate that UHRF1 is a primary factor in GC development, and suggest that understanding the molecular mechanisms underlying UHRF1 overexpression in GC may assist in discovering the underlying molecular mechanisms involved in GC tumorigenesis.

miR-146a and miR-146b are known to act as tumor suppressors in several types of tumor including GC and lung cancer (67-69). It has been demonstrated that UHRF1 is regulated by $\mathrm{miR}-146 \mathrm{a} / \mathrm{b}$ in GC (67). Furthermore, miR-146a/b overexpression significantly downregulated UHRF1 expression through directly targeting its 3'-untranslated region (UTR) (67). The downregulation was associated with DNA demethylation-dependent reactivation of a number of TSGs including Slit guidance ligand 3, cadherin 4 and $R U N X 3$, and a decrease in GC cell migration and metastasis (67). Furthermore, the downregulation of $\mathrm{miR}-146 \mathrm{a} / \mathrm{b}$ led to an increase in the expression of UHRF1, indicating that UHRF1 is negatively regulated by miR-146a/b in normal cells (67). A previous study also demonstrated that miR-146a binds to the 3'-UTR of UHRF1, providing additional evidence of a direct regulation of UHRF1 by miR-146a (65). Collectively, these results that support the hypothesis that UHRF1 mRNA is a direct target of miR-146a/b in GC, and miR-146a/b overexpression may be a promising strategy to downregulate UHRF1 in order to achieve GC metastasis inhibition through the reactivation of TSGs (Fig. 2A). 


\section{Regulation of UHRF1 by miRNAs in bladder cancer and kidney tumors}

Several previous studies have revealed the important role of UHRF1 in human bladder cancer (BC) invasion (1,70-72). UHRF1 was demonstrated to be overexpressed in bladder and kidney cancer, and its increased expression levels were associated with the stage and grading of BC (73). UHRF1 expression was increased in $\mathrm{BC}$ compared with matched normal tissues, and its overexpression was associated with tumor grade, relapse and survival rate (70). Several underlying molecular mechanisms have been suggested to explain the contribution of UHRF1 in the pathology of BC; however, the molecular mechanisms underlying UHRF1 regulation in this type of tumor are largely unknown. UHRF1 was demonstrated to promote the invasion of $\mathrm{BC}$ through epigenetic silencing of the tumor suppressor kisspeptin (KiSSl) and the regulator of G-protein signaling 2 (71,72). A recent study also reported that the overexpression of UHRF1 detected in BC tissues was accompanied by decreased levels of miR-124, acting as a tumor suppressor. Furthermore, miR-124 overexpression in human BC cells induced a decrease in UHRF1 expression, which led to the inhibition of cell proliferation, metastasis and invasion (74). Notably, a luciferase assay confirmed that miR-124 regulates UHRF1 expression by binding to the 3'-UTR of UHRF1, suggesting that UHRF1 overexpression observed in $\mathrm{BC}$ may be attributed at least in part to the loss of the expression of the tumor suppressor miR-124. The UHRF1 was recently demonstrated to be targeted in normal bladder cells by other tumor suppressor miRNAs including miR-145-5p and miR-145-3p (75). The downregulation of these miRNAs in BC causes overexpression of UHRF1 (75). Furthermore, this study demonstrated that BC expresses increased levels of UHRF1 compared with normal tissue, and its overexpression is associated with decreased levels of miR-145-5p and miR-145-3p. UHRF1 downregulation in BC cells induced a reactivation of a number of TSGs including PR domain zinc finger protein 2 (PRDM2), which acts as a histone methyltransferase on $\mathrm{H} 3 \mathrm{~K} 9$, and also led to the inhibition of several oncogenes including survivin (BIRC5) and centromere protein F (CENPF) (75-77). Notably, transfection of BC cells by miR-145-5p or miR-145-3p induced UHRF1 downregulation and consequently cell cycle arrest and apoptosis (75). Furthermore, luciferase reporter assays confirmed that miR-145-5p and miR-145-3p bind directly to different sites on 3'-UTR of UHRF1 (75). These results demonstrate that overexpression of miR-124, miR-145-5p and miR-145-3p in BC are able to decrease the expression levels of UHRF1, leading to $\mathrm{BC}$ metastasis inhibition by downregulating the anti-apoptotic proteins BIRC5 and CENPF (Fig. 2B). Collectively, these results support that miRNAs regulate TSG expression through the control of UHRF1, which dictates the level of DNA methylation and histone $\mathrm{H} 3$ methylation levels via PRDM2 and/or G9a.

UHRF1 was also revealed to be overexpressed in clear cell renal cell carcinoma (ccRCC), which represents $\sim 70 \%$ of all renal cell carcinomas (RCCs) $(73,78,79)$. UHRF1 overexpression at the mRNA and protein levels in ccRCC was associated with the downregulation of the TSG TP53 (79). This study demonstrated that UHRF1 directly binds to p53 protein causing ubiquitination-dependent degradation of p53, leading to apoptosis inhibition and ccRCC promotion (79). Recent studies have demonstrated that UHRF1 is overexpressed in primary and metastatic ccRCC tumors associated with decreased expression levels of miR-146a-5p, which has also been demonstrated to act as a tumor suppressor in non-small cell lung cancer (NSCLC) and prostate cancer $(78,80,81)$. Notably, overexpression of miR-146a-5p in two different human kidney cancer cell lines (786-O and ACHN) significantly decreased the expression of UHRF1 (78). These results indicate that miR-146a-5p negatively regulates the expression of UHRF1 in ccRCC, and miR-146a-5p upregulation is sufficient to induce UHRF1 degradation and $\mathrm{p} 53$ reactivation leading to the inhibition of ccRCC progression (Fig. 2C). The overexpression of miR-101, a tumor suppressor miRNA, was recently demonstrated to inhibit the expression of UHRF1 in RCC (82-84). Currently, it is not yet known whether UHRF1 is regulated by a unique miRNA in a cell-specific manner, or by several miRNAs within a same cell, and therefore further investigation is required.

\section{Regulation of UHRF1 by miR-9 in colorectal cancer}

Several previous studies have demonstrated that UHRF1 is overexpressed in colorectal cancer (CRC) and induces epigenetic silencing of several TSGs including $p 16^{I N K 4 A}$ leading to cell growth and metastasis $(39,85,86)$. CRC tissues and cell lines have exhibited overexpression of UHRF1 with decreased levels of p16 ${ }^{\mathrm{INK} 4 \mathrm{~A}}$ expression and increased metastasis (39). Conversely, UHRF1 downregulation induced an upregulation of p16 ${ }^{\text {INK4A }}$, cell proliferation, migration inhibition, cell cycle arrest and apoptosis (39). In the same context, increased levels of UHRF1 were detected in specimens of CRC, and in vitro UHRF1 downregulation resulted in inhibition of CRC proliferation (86). Collectively, these studies indicate that UHRF1 overexpression may be a primary event in CRC development, and therefore its targeting may represent a novel approach to CRC therapy. However, the upstream factors which regulate UHRF1 expression in CRC remain unclear.

UHRF1 overexpression was associated with decreased survival rates of patients with CRC, and a decrease in the expression of the tumor suppressor miR-9 (87-90). The luciferase assay demonstrated that UHRF1 is directly regulated by miR-9, indicating that UHRF1 overexpression in CRC results from a decrease in miR-9 expression (87). Notably, miR-9 overexpression in CRC cells was able to significantly decrease UHRF1 expression and cell proliferation, and induce apoptosis (Fig. 2D). These results demonstrate that another miRNA is involved in CRC compared with the aforementioned types of cancer. Thus, these results suggest a cell-specific dependence of UHRF1 regulation towards miRNAs.

\section{Regulation of UHRF1 by miR-193a-3p in NSCLC}

UHRF1 was also identified as being overexpressed in several other types of NSCLC, and may serve as a diagnostic and therapeutic marker for this type of cancer (91-93). Increased expression levels of UHRF1 were identified in primary NSCLC accompanied with increased levels of the three DNA methyltransferases DNMT1, DNMT3A and 
DNMT3B concomitantly with hypermethylation of several TSG promoters including cyclin-dependent kinase inhibitor 2 $(C D K N 2 A)$ and Ras-associated domain-containing protein 1 (RASSF1) (94). Notably, UHRF1 depletion induced a promoter demethylation-dependent reactivation of $C D K N 2 A$ and $R A S S F 1$ with subsequent inhibition of cell proliferation and metastasis (94). These results suggest that UHRF1 overexpression is involved in the molecular pathogenesis of NSCLC and that the upstream regulatory mechanisms begin to be elucidated. Accordingly, miR-193a-3p serves as a tumor suppressor in cancer, and its overexpression was demonstrated to repress NSCLC progression (95). Recently, it has been identified that miR-193a-3p inhibits NSCLC metastasis by downregulating several oncogenes including UHRF1 (Fig. 2E), suggesting that UHRF1 expression is inversely associated with miR-193a-3p in NSCLC (93). Furthermore, these studies collectively support the hypothesis that each type of cancer has a different type of UHRF1 in terms of miRNA.

\section{Conclusion}

The present review provides an insight into the molecular mechanism underlying how miRNAs may function as tumor suppressors by directly inhibiting the expression of the oncogene UHRF1 which is considered to be a master of the epigenetic silencing of several TSGs in cancer. It is also indicated that the combined expression of regulator miRNA and UHRF1 may be a potential diagnostic and prognostic marker in cancer. UHRF1 is overexpressed in several types of human cancer, which contributes to the increase in cell proliferation, metastasis and the inhibition of apoptosis. Considering the fact that abnormalities in miRNA expression may be a potent cause of cancer development, exploring the direct association between UHRF1 and miRNAs will increase our understanding of tumor pathology, and may also allow the development of novel therapeutic strategies based on specific targeting of the miRNA/UHRF1 pathways in several types of tumor (Fig. 2). However, future investigations are required to understand the downregulation of miRNAs in the pathogenesis of cancer. Furthermore, the assessment of the cell-specific miRNA-dependent regulation of UHRF1 as well as the origin of the deregulation of these types of miRNA may also be required.

\section{Acknowledgements}

The present review was supported by a French State fund managed by the Agence Nationale de la Recherche under the framework program Investissements d'Avenir ANR-10-IDEX-0002-02 (grant no. ANR-10-LABX-0030-INRT).

\section{References}

1. Unoki M, Nishidate T and Nakamura Y: ICBP90, an E2F-1 target, recruits $\mathrm{HDAC} 1$ and binds to methyl-CpG through its SRA domain. Oncogene 23: 7601-7610, 2004.

2. Jin W, Chen L, Chen Y, Xu SG, Di GH, Yin WJ, Wu J and Shao ZM: UHRF1 is associated with epigenetic silencing of BRCA1 in sporadic breast cancer. Breast Cancer Res Treat 123: $359-373,2010$
3. Achour M, Jacq X, Rondé P, Alhosin M, Charlot C, Chataigneau T, Jeanblanc M, Macaluso M, Giordano A, Hughes AD, et al: The interaction of the SRA domain of ICBP90 with a novel domain of DNMT1 is involved in the regulation of VEGF gene expression. Oncogene 27: 2187-2197, 2008.

4. Bronner C, Achour M, Arima Y, Chataigneau T, Saya H and Schini-Kerth VB: The UHRF family: Oncogenes that are drugable targets for cancer therapy in the near future? Pharmacol Ther 115: 419-434, 2007.

5. Berkyurek AC, Suetake I, Arita K, Takeshita K, Nakagawa A, Shirakawa M and Tajima S: The DNA methyltransferase Dnmt1 directly interacts with the SET and RING finger-associated (SRA) domain of the multifunctional protein Uhrf1 to facilitate accession of the catalytic center to hemi-methylated DNA. J Biol Chemistry 289: 379-386, 2014.

6. Alhosin M, Omran Z, Zamzami MA, Al-Malki AL, Choudhry H, Mousli M and Bronner C: Signalling pathways in UHRF1-dependent regulation of tumor suppressor genes in cancer. J Exp Clin Cancer Res 35: 174, 2016.

7. Bronner C, Krifa M and Mousli M: Increasing role of UHRF1 in the reading and inheritance of the epigenetic code as well as in tumorogenesis. Biochem Pharmacol 86: 1643-1649, 2013.

8. Alhosin M, Sharif T, Mousli M, Etienne-Selloum N,Fuhrmann G, Schini-Kerth VB and Bronner C: Down-regulation of UHRF1, associated with re-expression of tumor suppressor genes, is a common feature of natural compounds exhibiting anti-cancer properties. J Exp Clin Cancer Res 30: 41, 2011.

9. Bronner C, Chataigneau T, Schini-Kerth VB and Landry Y: The 'Epigenetic Code Replication Machinery', ECREM: A promising drugable target of the epigenetic cell memory. Curr Med Chemistry 14: 2629-2641, 2007.

10. Liu X, Gao Q, Li P, Zhao Q, Zhang J, Li J, Koseki H and Wong J: UHRF1 targets DNMT1 for DNA methylation through cooperative binding of hemi-methylated DNA and methylated H3K9. Nat Commun 4: 1563, 2013.

11. Nady N, Lemak A, Walker JR, Avvakumov GV, Kareta MS, Achour M, Xue S, Duan S, Allali-Hassani A, Zuo X, et al: Recognition of multivalent histone states associated with heterochromatin by UHRF1 protein. J Biol Chem 286: 24300-24311, 2011.

12. Karagianni P, Amazit L, Qin J and Wong J: ICBP90, a novel methyl $\mathrm{K} 9 \mathrm{H} 3$ binding protein linking protein ubiquitination with heterochromatin formation. Mol Cell Biol 28: 705-717, 2008.

13. Papait R, Pistore C, Grazini U, Babbio F, Cogliati S, Pecoraro D, Brino L, Morand AL, Dechampesme AM, Spada F, et al: The PHD domain of Np95 (mUHRF1) is involved in large-scale reorganization of pericentromeric heterochromatin. Mol Biol Cell 19: 3554-3563, 2008.

14. Jenkins Y, Markovtsov V, Lang W, Sharma P, Pearsall D, Warner J, Franci C, Huang B, Huang J, Yam GC, et al: Critical role of the ubiquitin ligase activity of UHRF1, a nuclear RING finger protein, in tumor cell growth. Mol Biol Cell 16: 5621-5629, 2005.

15. Citterio E, Papait R, Nicassio F, Vecchi M, Gomiero P, Mantovani R, Di Fiore PP and Bonapace IM: Np95 is a histone-binding protein endowed with ubiquitin ligase activity. Mol Cell Biol 24: 2526-2535, 2004.

16. Li M, Chen D, Shiloh A, Luo J, Nikolaev AY, Qin J and Gu W: Deubiquitination of $\mathrm{p} 53$ by HAUSP is an important pathway for p53 stabilization. Nature 416: 648-653, 2002.

17. Qin W, Leonhardt H and Spada F: Usp7 and Uhrf1 control ubiquitination and stability of the maintenance DNA methyltransferase Dnmt1. J Cell Biochem 112: 439-444, 2011.

18. Felle M, Joppien S, Németh A, Diermeier S, Thalhammer V, Dobner T, Kremmer E, Kappler R and Längst G: The USP7/ Dnmtl complex stimulates the DNA methylation activity of Dnmt1 and regulates the stability of UHRF1. Nucleic Acids Res 39: 8355-8365, 2011.

19. Ma H, Chen H, Guo X, Wang Z, Sowa ME, Zheng L, Hu S, Zeng P, Guo R, Diao J, et al: M phase phosphorylation of the epigenetic regulator UHRF1 regulates its physical association with the deubiquitylase USP7 and stability. Proc Natl Acad Sci USA 109: 4828-4833, 2012.

20. Arita K, Ariyoshi M, Tochio H, Nakamura Y and Shirakawa M: Recognition of hemi-methylated DNA by the SRA protein UHRF1 by a base-flipping mechanism. Nature 455: 818-821, 2008.

21. Avvakumov GV, Walker JR, Xue S, Li Y, Duan S, Bronner C, Arrowsmith $\mathrm{CH}$ and Dhe-Paganon S: Structural basis for recognition of hemi-methylated DNA by the SRA domain of human UHRF1. Nature 455: 822-825, 2008. 
22. Bostick M, Kim JK, Estève PO, Clark A, Pradhan S and Jacobsen SE: UHRF1 plays a role in maintaining DNA methylation in mammalian cells. Science 317: 1760-1764, 2007.

23. Unoki M, Brunet $\mathbf{J}$ and Mousli M: Drug discovery targeting epigenetic codes: The great potential of UHRF1, which links DNA methylation and histone modifications, as a drug target in cancers and toxoplasmosis. Biochem Pharmacol 78: 1279-1288, 2009.

24. Hervouet E, Lalier L, Debien E, Cheray M, Geairon A, Rogniaux H, Loussouarn D, Martin SA, Vallette FM and Cartron PF: Disruption of Dnmt1/PCNA/UHRF1 interactions promotes tumorigenesis from human and mice glial cells. PLoS One 5: e11333, 2010.

25. Pacaud R, Brocard E, Lalier L, Hervouet E, Vallette FM and Cartron PF: The DNMT1/PCNA/UHRF1 disruption induces tumorigenesis characterized by similar genetic and epigenetic signatures. Sci Reports 4: 4230, 2014

26. Ge TT, Yang M, Chen Z, Lou G and Gu T: UHRF1 gene silencing inhibits cell proliferation and promotes cell apoptosis in human cervical squamous cell carcinoma CaSki cells. J Ovarian Res 9: $42,2016$.

27. Wan X, Yang S, Huang W, Wu D, Chen H, Wu M, Li J, Li T and $\mathrm{Li}$ Y: UHRF1 overexpression is involved in cell proliferation and biochemical recurrence in prostate cancer after radical prostatectomy. J Exp Clin Cancer Res 35: 34, 2016.

28. Abu-Alainin W, Gana T, Liloglou T, Olayanju A, Barrera LN, Ferguson R, Campbell F, Andrews T, Goldring C, Kitteringham N, et al: UHRF1 regulation of the Keap1-Nrf2 pathway in pancreatic cancer contributes to oncogenesis J Pathology 238: 423-433, 2016.

29. UHRF1 is an oncogene that promotes DNA hypomethylation. Cancer Discov 4: OF9, 2014.

30. Guan D, Factor D, Liu Y, Wang Z and Kao HY: The epigenetic regulator UHRF1 promotes ubiquitination-mediated degradation of the tumor-suppressor protein promyelocytic leukemia protein. Oncogene 32: 3819-3828, 2013.

31. Babbio F, Pistore C, Curti L, Castiglioni I, Kunderfranco P, Brino L, Oudet P, Seiler R, Thalman GN, Roggero E, et al: The SRA protein UHRF1 promotes epigenetic crosstalks and is involved in prostate cancer progression. Oncogene 31 : 4878-4887, 2012.

32. Zhou L, Shang Y, Jin Z, Zhang W, Lv C, Zhao X, Liu Y, Li N and Liang J: UHRF1 promotes proliferation of gastric cancer via mediating tumor suppressor gene hypermethylation. Cancer Biol Ther 16: 1241-1251, 2015.

33. Qu X, Davison J, Du L, Storer B, Stirewalt DL, Heimfeld S, Estey E, Appelbaum FR and Fang M: Identification of differentially methylated markers among cytogenetic risk groups of acute myeloid leukemia. Epigenetics 10: 526-535, 2015.

34. Kim JH, Shim JW, Eum DY, Kim SD, Choi SH, Yang K, Heo K and Park MT: Downregulation of UHRF1 increases tumor malignancy by activating the CXCR4/AKT-JNK/IL-6/Snail signaling axis in hepatocellular carcinoma cells. Sci Rep 7: 2798, 2017.

35. Jung YD, Shim JW, Park SJ, Choi SH, Yang K, Heo K and Park MT: Downregulation of UHRF1 promotes EMT via inducing CXCR4 in human cancer cells. Int J Oncol 46: 1232-1242, 2015.

36. Ashraf W, Ibrahim A, Alhosin M, Zaayter L, Ouararhni K, Papin C, Ahmad T, Hamiche A, Mély Y, Bronner C and Mousli M: The epigenetic integrator UHRF1: On the road to become a universal biomarker for cancer. Oncotarget 8: 51946-51962, 2017.

37. Cui L, Chen J, Zhang Q, Wang X, Qu J, Zhang J and Dang S: Up-regulation of UHRF1 by oncogenic Ras promoted the growth, migration, and metastasis of pancreatic cancer cells. Mol Cell Biochem 400: 223-232, 2015.

38. Qin L, Dong Z and Zhang JT: Reversible epigenetic regulation of 14-3-3sigma expression in acquired gemcitabine resistance by uhrf1 and DNA methyltransferase 1. Mol Pharmacol 86: 561-569, 2014.

39. Wang F, Yang YZ, Shi CZ, Zhang P, Moyer MP, Zhang HZ, Zou Y and Qin HL: UHRF1 promotes cell growth and metastasis through repression of p16(ink(4)a) in colorectal cancer. Ann Surg Oncol 19: 2753-2762, 2012

40. Achour M, Mousli M, Alhosin M, Ibrahim A, Peluso J, Muller CD, Schini-Kerth VB, Hamiche A, Dhe-Paganon S and Bronner C: Epigallocatechin-3-gallate up-regulates tumor suppressor gene expression via a reactive oxygen species-dependent down-regulation of UHRF1. Biochem Biophys Res Commun 430: 208-212, 2013.

41. Djebali S, Davis CA, Merkel A, Dobin A, Lassmann T, Mortazavi A, Tanzer A, Lagarde J, Lin W, Schlesinger F, et al: Landscape of transcription in human cells. Nature 489: 101-108, 2012.
42. Choudhry H, Harris AL and McIntyre A: The tumour hypoxia induced non-coding transcriptome. Mol Aspects Med 47-48: 35-53, 2016.

43. Choudhry H and Mole DR: Hypoxic regulation of the noncoding genome and NEAT1. Brief Funct Genomics 15: 174-185, 2016.

44. Josse C and Bours V: MicroRNAs and inflammation in colorectal cancer. Adv Exp Med Biol 937: 53-69, 2016.

45. Vicente R, Noël D, Pers YM, Apparailly F and Jorgensen C: Deregulation and therapeutic potential of microRNAs in arthritic diseases. Nat Rev Rheumatol 12: 496, 2016.

46. Guedes JR, Santana I, Cunha C, Duro D, Almeida MR, Cardoso AM, de Lima MC and Cardoso AL: MicroRNA deregulation and chemotaxis and phagocytosis impairment in Alzheimer's disease. Alzheimers Dement (Amst) 3: 7-17, 2015.

47. Irmak-Yazicioglu MB: Mechanisms of MicroRNA Deregulation and MicroRNA Targets in Gastric Cancer. Oncol Res Treat 39: 136-139, 2016.

48. Lujambio A and Lowe SW: The microcosmos of cancer. Nature 482: 347-355, 2012

49. Ruan X, Zuo Q, Jia H, Chau J, Lin J, Ao J, Xia X, Liu H, Habib SL, Fu C and Li B: P53 deficiency-induced Smad1 upregulation suppresses tumorigenesis and causes chemoresistance in colorectal cancers. J Mol Cell Biol 7: 105-118, 2015.

50. Shah MY, Ferrajoli A, Sood AK, Lopez-Berestein G and Calin GA: microRNA therapeutics in cancer-an emerging concept. EBioMedicine 12: 34-42, 2016.

51. Li G, Yang F, Gu S, Li Z and Xue M: MicroRNA-101 induces apoptosis in cisplatin-resistant gastric cancer cells by targeting VEGF-C. Mol Med Rep 13: 572-578, 2016.

52. Hayes J, Peruzzi PP and Lawler S: MicroRNAs in cancer: Biomarkers, functions and therapy. Trends Mol Med 20: 460-469, 2014.

53. Xu J, Wang Z, Li S, Chen J, Zhang J, Jiang C, Zhao Z, Li J, Li Y and Li X: Combinatorial epigenetic regulation of non-coding RNAs has profound effects on oncogenic pathways in breast cancer subtypes. Brief Bioinform: Oct 14, 2016 (Epub ahead of print).

54. Kaur S, Lotsari-Salomaa JE, Seppänen-Kaijansinkko R and Peltomäki P: MicroRNA Methylation in Colorectal Cancer. Adv Exp Med Biol 937: 109-122, 2016.

55. Voorhoeve PM: MicroRNAs: Oncogenes, tumor suppressors or master regulators of cancer heterogeneity? Biochim Biophys Acta 1805: 72-86, 2010

56. Zhou X, Wang X, Huang Z, Wang J, Zhu W, Shu Y and Liu P: Prognostic value of miR-21 in various cancers: An updating meta-analysis. PLoS One 9: e102413, 2014.

57. Medina PP, Nolde $M$ and Slack FJ: OncomiR addiction in an in vivo model of microRNA-21-induced pre-B-cell lymphoma. Nature 467: 86-90, 2010.

58. Selcuklu SD, Donoghue MT and Spillane C: miR-21 as a key regulator of oncogenic processes. Biochem Soc Transac 37: 918-925, 2009.

59. Favreau AJ, McGlauflin RE, Duarte CW and Sathyanarayana P: miR-199b, a novel tumor suppressor miRNA in acute myeloid leukemia with prognostic implications. Exp Hematol Oncol 5: 4, 2016.

60. Venkataraman S, Birks DK, Balakrishnan I, Alimova I, Harris PS, Patel PR, Handler MH, Dubuc A, Taylor MD, Foreman NK and Vibhakar R: MicroRNA 218 acts as a tumor suppressor by targeting multiple cancer phenotype-associated genes in medulloblastoma. J Biol Chem 288: 1918-1928, 2013.

61. Boukhari A, Alhosin M, Bronner C, Sagini K, Truchot C, Sick E, Schini-Kerth VB, André P, Mély Y, Mousli M and Gies JP: CD47 activation-induced UHRF1 over-expression is associated with silencing of tumor suppressor gene p16INK4A in glioblastoma cells. Anticancer Res 35: 149-157, 2015.

62. Arima Y, Hirota T, Bronner C, Mousli M, Fujiwara T, Niwa S, Ishikawa $\mathrm{H}$ and Saya $\mathrm{H}$ : Down-regulation of nuclear protein ICBP90 by p53/p21Cip1/WAF1-dependent DNA-damage checkpoint signals contributes to cell cycle arrest at G1/S transition. Genes Cells 9: 131-142, 2004.

63. Alhosin M, Abusnina A, Achour M, Sharif T, Muller C, Peluso J, Chataigneau T, Lugnier C, Schini-Kerth VB, Bronner C and Fuhrmann G: Induction of apoptosis by thymoquinone in lymphoblastic leukemia Jurkat cells is mediated by a p73-dependent pathway which targets the epigenetic integrator UHRF1. Biochem Pharmacol 79: 1251-1260, 2010.

64. Wu SM, Cheng WL, Liao CJ, Chi HC, Lin YH, Tseng YH, Tsai CY, Chen CY, Lin SL, Chen WJ, et al: Negative modulation of the epigenetic regulator, UHRF1, by thyroid hormone receptors suppresses liver cancer cell growth. Int J Cancer 137: 37-49, 2015. 
65. Soleimani A, Ghanadi K, Noormohammadi Z and Irani S: The correlation between miR-146a C/G polymorphism and UHRF1gene expression level in gastric tumor. J Dig Dis 17: $169-174,2016$

66. Ge M, Gui Z, Wang X and Yan F: Analysis of the UHRF1 expression in serum and tissue for gastric cancer detection. Biomarkers 20: 183-188, 2015.

67. Zhou L, Zhao X, Han Y, Lu Y, Shang Y, Liu C, Li T, Jin Z, Fan D and $\mathrm{Wu} \mathrm{K}$ : Regulation of UHRF1 by miR-146a/b modulates gastric cancer invasion and metastasis. FASEB J 27: 4929-4939, 2013.

68. Patnaik SK, Kannisto E, Mallick R and Yendamuri S: Overexpression of the lung cancer-prognostic miR-146b microRNAs has a minimal and negative effect on the malignant phenotype of A549 lung cancer cells. PLoS One 6: e22379, 2011

69. Xiao B, Zhu ED, Li N, Lu DS, Li W, Li BS, Zhao YL, Mao XH, Guo G, Yu PW and Zou QM: Increased miR-146a in gastric cancer directly targets SMAD4 and is involved in modulating cell proliferation and apoptosis. Oncol Rep 27: 559-566, 2012.

70. Yang GL, Zhang LH, Bo JJ, Chen HG, Cao M, Liu DM and Huang YR: UHRF1 is associated with tumor recurrence in non-muscle-invasive bladder cancer. Med Oncol 29: 842-847, 2012.

71. Zhang Y, Huang Z, Zhu Z, Zheng X, Liu J, Han Z, Ma X and Zhang Y: Upregulated UHRF1 promotes bladder cancer cell invasion by epigenetic silencing of KiSS1. PLoS One 9: e104252, 2014.

72. Ying L, Lin J, Qiu F, Cao M, Chen H, Liu Z and Huang Y: Epigenetic repression of regulator of G-protein signaling 2 by ubiquitin-like with PHD and ring-finger domain 1 promotes bladder cancer progression. FEBS J 282: 174-182, 2015.

73. Unoki M, Kelly JD, Neal DE, Ponder BA, Nakamura Y and Hamamoto R: UHRF1 is a novel molecular marker for diagnosis and the prognosis of bladder cancer. Br J Cancer 101: 98-105, 2009.

74. Wang X, Wu Q, Xu B, Wang P, Fan W, Cai Y, Gu X and Meng F: miR-124 exerts tumor suppressive functions on the cell proliferation, motility and angiogenesis of bladder cancer by fine-tuning UHRF1. FEBS J 282: 4376-4388, 2015.

75. Matsushita R, Yoshino H, Enokida H, Goto Y, Miyamoto K, Yonemori M, Inoguchi S, Nakagawa M and Seki N: Regulation of UHRF1 by dual-strand tumor-suppressor microRNA-145 (miR-145-5p and miR-145-3p): Inhibition of bladder cancer cell aggressiveness. Oncotarget 7: 28460-28487, 2016.

76. Xie W, Li X, Chen X, Huang S and Huang S: Decreased expression of PRDM2 (RIZ1) and its correlation with risk stratification in patients with myelodysplastic syndrome. Br J Haematol 150: 242-244, 2010

77. Kim KC, Geng L and Huang S: Inactivation of a histone methyltransferase by mutations in human cancers. Cancer Res 63: 7619-7623, 2003

78. Wotschofsky Z, Gummlich L, Liep J, Stephan C, Kilic E, Jung K, Billaud JN and Meyer HA: Integrated microRNA and mRNA signature associated with the transition from the locally confined to the metastasized clear cell renal cell carcinoma exemplified by miR-146-5p. PLoS One 11: e0148746, 2016.

79. Ma J, Peng J, Mo R, Ma S, Wang J, Zang L, Li W and Fan J: Ubiquitin E3 ligase UHRF1 regulates p53 ubiquitination and p53-dependent cell apoptosis in clear cell renal cell carcinoma. Biochem Biophys Res Commun 464: 147-153, 2015.

80. Li YL, Wang J, Zhang CY, Shen YQ, Wang HM, Ding L, Gu YC Lou JT, Zhao XT, Ma Z and Jin YX: MiR-146a-5p inhibits cell proliferation and cell cycle progression in NSCLC cell lines by targeting CCND1 and CCND2. Oncotarget 7: 59287-59298, 2016.

81. Sun Q, Zhao X, Liu X, Wang Y, Huang J, Jiang B, Chen Q and $\mathrm{Yu}$ J: miR-146a functions as a tumor suppressor in prostate cancer by targeting Rac1. Prostate 74: 1613-1621, 2014.
82. Riquelme I, Tapia O, Leal P, Sandoval A, Varga MG, Letelier P, Buchegger K, Bizama C, Espinoza JA, Peek RM, et al: miR-101-2, miR-125b-2 and miR-451a act as potential tumor suppressors in gastric cancer through regulation of the PI3K/AKT/mTOR pathway. Cell Oncology (Dordr) 39: 23-33, 2016.

83. Farhadi E, Zaker F, Safa M and Rezvani MR: miR-101 sensitizes K562 cell line to imatinib through Jak2 downregulation and inhibition of NF- $\mathrm{\kappa B}$ target genes. Tumour Biol 37: 14117-14128, 2016.

84. Goto Y, Kurozumi A, Nohata N, Kojima S, Matsushita R, Yoshino H, Yamazaki K, Ishida Y, Ichikawa T, Naya Y and Seki N: The microRNA signature of patients with sunitinib failure: Regulation of UHRF1 pathways by microRNA-101 in renal cell carcinoma. Oncotarget 7: 59070-59086, 2016.

85. Walter A, Etienne-Selloum N, Brasse D, Khallouf H, Bronner C, Rio MC, Beretz A and Schini-Kerth VB: Intake of grape-derived polyphenols reduces C26 tumor growth by inhibiting angiogenesis and inducing apoptosis. FASEB J 24: 3360-3369, 2010

86. Kofunato Y, Kumamoto K, Saitou K, Hayase S, Okayama H, Miyamoto K, Sato Y, Katakura K, Nakamura I, Ohki S, et al: UHRF1 expression is upregulated and associated with cellular proliferation in colorectal cancer. Oncol Rep 28: 1997-2002, 2012.

87. Zhu M, Xu Y, Ge M, Gui Z and Yan F: Regulation of UHRF1 by microRNA-9 modulates colorectal cancer cell proliferation and apoptosis. Cancer Sci 106: 833-839, 2015.

88. Bandres E, Agirre X, Bitarte N, Ramirez N, Zarate R, Roman-Gomez J, Prosper F and Garcia-Foncillas J: Epigenetic regulation of microRNA expression in colorectal cancer. Int $\mathrm{J}$ Cancer 125: 2737-2743, 2009.

89. Cekaite L, Rantala JK, Bruun J, Guriby M, Agesen TH, Danielsen SA, Lind GE, Nesbakken A, Kallioniemi O, Lothe RA and Skotheim RI: MiR-9, -31, and -182 deregulation promote proliferation and tumor cell survival in colon cancer. Neoplasia 14: 868-879, 2012.

90. Lu MH, Huang CC, Pan MR, Chen HH and Hung WC: Prospero homeobox 1 promotes epithelial-mesenchymal transition in colon cancer cells by inhibiting E-cadherin via miR-9. Clin Cancer Res 18: 6416-6425, 2012

91. Unoki M, Daigo Y, Koinuma J, Tsuchiya E, Hamamoto R and Nakamura Y: UHRF1 is a novel diagnostic marker of lung cancer. Br J Cancer 103: 217-222, 2010.

92. Wu H, Meng S, Xu Q, Wang X, Wang J, Gong R, Song Y, Duan Y and Zhang Y: Gene expression profiling of lung adenocarcinoma in Xuanwei, China. Eur J Cancer Prev 25: 508-517, 2016.

93. Deng W, Yan M, Yu T, Ge H, Lin H, Li J, Liu Y, Geng Q, Zhu M, Liu L, et al: Quantitative proteomic analysis of the metastasis-inhibitory mechanism of miR-193a-3p in non-small cell lung cancer. Cell Physiol Biochem 35: 1677-1688, 2015.

94. Daskalos A, Oleksiewicz U, Filia A, Nikolaidis G, Xinarianos G, Gosney JR, Malliri A, Field JK and Liloglou T: UHRF1-mediated tumor suppressor gene inactivation in nonsmall cell lung cancer. Cancer 117: 1027-1037, 2011

95. Yu T, Li J, Yan M, Liu L, Lin H, Zhao F, Sun L, Zhang Y, Cui Y, Zhang F, et al: MicroRNA-193a-3p and -5p suppress the metastasis of human non-small-cell lung cancer by downregulating the ERBB4/PIK3R3/mTOR/S6K2 signaling pathway. Oncogene 34: 413-423, 2015

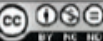

This work is licensed under a Creative Commons Attribution-NonCommercial-NoDerivatives 4.0 International (CC BY-NC-ND 4.0) License. 